\title{
Evaluations of $k$-fold Euler/Zagier sums: a compendium of results for arbitrary $k$
}

\author{
J. M. Borwein jborwein@cecm.sfu.ca \\ D. M. Bradley dbradley@cecm.sfu.ca \\ CECM, Simon Fraser University, Burnaby, B.C. V5A 1S6, Canada \\ http://www.cecm.sfu.ca/ \\ D. J. Broadhurst D.Broadhurst@open.ac.uk \\ Physics Department, Open University, Milton Keynes MK7 6AA, UK \\ http://yan.open.ac.uk/
}

Submitted: September 2, 1996; Accepted: October 31, 1996.

\begin{abstract}
Euler sums (also called Zagier sums) occur within the context of knot theory and quantum field theory. There are various conjectures related to these sums whose incompletion is a sign that both the mathematics and physics communities do not yet completely understand the field. Here, we assemble results for Euler/Zagier sums (also known as multidimensional zeta/harmonic sums) of arbitrary depth, including sign alternations. Many of our results were obtained empirically and are apparently new. By carefully compiling and examining a huge data base of high precision numerical evaluations, we can claim with some confidence that certain classes of results are exhaustive. While many proofs are lacking, we have sketched derivations of all results that have so far been proved.
\end{abstract}


THE ElECTRONiC JOURNAL OF COMBINATORICS 4 (No.2) (1997), \#R5

\section{Introduction}

We consider $k$-fold Euler sums $[13,2,3]$ (also called Zagier sums) of arbitrary depth $k$. These sums occur in a natural way within the context of knot theory and quantum field theory (see [4] for an extended bibliography), carrying on a rich tradition of algebra and number theory as pioneered by Euler. There are various conjectures related to these sums (see e.g. (8) below) whose incompletion is a sign that both the mathematics and physics communities do not yet completely understand the field, whence new results are welcome.

As in [4] we allow for all possible alternations of signs, with $\sigma_{j}= \pm 1$ in

$$
\zeta\left(s_{1}, \ldots, s_{k} ; \sigma_{1}, \ldots, \sigma_{k}\right)=\sum_{n_{j}>n_{j+1}>0} \prod_{j=1}^{k} \frac{\sigma_{j}^{n_{j}}}{n_{j}^{s_{j}}},
$$

since alternating Euler sums are essential [7] to the connection [18] of knot theory with quantum field theory $[8,6]$. The integral representation

$$
\begin{aligned}
\zeta\left(s_{1}, \ldots, s_{k} ; \sigma_{1}, \ldots, \sigma_{k}\right) & =\prod_{j=1}^{k} \frac{1}{\Gamma\left(s_{j}\right)} \int_{1}^{\infty} \frac{d y_{j}}{y_{j}} \frac{\left(\ln y_{j}\right)^{s_{j}-1}}{\prod_{i=1}^{j} \sigma_{i} y_{i}-1}, \\
& =\prod_{j=1}^{k} \frac{1}{\Gamma\left(s_{j}\right)} \int_{0}^{\infty} \frac{u_{j}^{s_{j}-1} d u_{j}}{\tau_{j} \exp \left(\sum_{i=1}^{j} u_{i}\right)-1}
\end{aligned}
$$

generalizes that given in [10] for non-alternating sums. Here,

$$
\tau_{j}:=\prod_{i=1}^{j} \sigma_{i}
$$

For positive integers $s_{j}$, each $\left(\ln y_{j}\right)^{s_{j}-1} / \Gamma\left(s_{j}\right)$ in the integrand of $(2)$ can be written as an iterated integral of the product $x_{1}^{-1} d x_{1} \cdots x_{s_{j}}^{-1} d x_{s_{j}}$. Thus, we have the alternative $\left(s_{1}+s_{2}+\cdots+s_{k}\right)$-dimensional iterated-integral representation

$$
\zeta\left(s_{1}, \ldots, s_{k} ; \sigma_{1}, \ldots, \sigma_{k}\right)=\int_{0}^{1} \Omega^{s_{1}-1} \omega_{1} \Omega^{s_{2}-1} \omega_{2} \cdots \Omega^{s_{k}-1} \omega_{k}, \quad s_{1}>1,
$$

in which the integrand denotes a string of distinct differential 1 -forms of type $\Omega=d x / x$ and $\omega_{j}$ is given by

$$
\omega_{j}:=\frac{\tau_{j} d x_{j}}{1-x_{j} \tau_{j}} .
$$


Note that (5) shows that Euler sums form a ring, with a product of sums given by ternary reshutfles of the 1 -forms $d x / x, d x /(1-x)$, and $d x /(1+x)$, just as products of non-alternating sums involve binary $[17,21]$ reshuffles of $d x / x$ and $d x /(1-x)$.

We shall combine the strings of exponents and signs into a single string, with $s_{j}$ in the $j$ th position when $\sigma_{j}=+1$, and $\bar{s}_{j}$ in the $j$ th position when $\sigma_{j}=-1$. We denote $n$ repetitions of a substring by $\{\ldots\}_{n}$. Finally, we are obliged to point out that the notation (1) is not completely standard. In [10], for example, the argument list is reversed. Unfortunately, both notations have proliferated.

For non-alternating sums, several results are known, notably the duality relation [17]

$$
\zeta\left(m_{1}+2,\{1\}_{n_{1}}, \ldots, m_{p}+2,\{1\}_{n_{p}}\right)=\zeta\left(n_{p}+2,\{1\}_{m_{p}}, \ldots, n_{1}+2,\{1\}_{m_{1}}\right),
$$

an explicit evaluation ${ }^{1}$ of the self-dual case with $m_{j}=n_{j}=1$, by Zagier [21, 22], (also cited in [10]]:

and the sum rule [14]:

$$
\zeta\left(\{3,1\}_{n}\right) \stackrel{?}{=} \frac{2 \cdot \pi^{4 n}}{(4 n+2) !}
$$

$$
\sum_{\substack{n_{j}>\delta_{j, 1} \\ N=\Sigma_{j} n_{j}}} \zeta\left(n_{1}, n_{2}, \ldots, n_{k}\right)=\zeta(N)
$$

These, and other results have been recast in the language of graded commutative rings [16].

We find that (8) is the first member of a class of arbitrary-depth results for selfdual non-alternating sums that evaluate to rational multiples of powers of $\pi^{2}$, and that alternating Euler sums of arbitrary depth have a comparably rich structure.

\section{Generating functions and relations}

We derived the generating function

$$
\sum_{m, n \geq 0} x^{m+1} y^{n+1} \zeta\left(m+2,\{1\}_{n}\right)=1-\exp \left\{\sum_{k \geq 2} \frac{x^{k}+y^{k}-(x+y)^{k}}{k} \zeta(k)\right\},
$$

${ }^{1}$ We mark with $\stackrel{?}{=}$ conjectures for which we have overwhelming evidence, but no proof. For unmarked equalities, we either cite proofs from the literature, or provide a proof sketch in the appendix. 
THE ELECTROniC Journal of COMBinatorics 4 (No.2) (1997), \#R5

for the non-alternating sums in the $p=1$ case of (7), and the generators

$$
\begin{aligned}
\sum_{n \geq 0} x^{s n} \zeta\left(\{s\}_{n}\right) & =\prod_{j \geq 1}\left(1+\frac{x^{s}}{j^{s}}\right)=\exp \left\{\sum_{k \geq 1} \frac{(-1)^{k-1} x^{s k} \zeta(s k)}{k}\right\}, \\
\sum_{n \geq 0} x^{s n} \zeta\left(\{\bar{s}\}_{n}\right) & =\prod_{j \geq 1}\left(1+(-1)^{j} \frac{x^{s}}{j^{s}}\right) \\
& =\exp \left\{\sum_{k \geq 1}\left(\frac{2(x / 2)^{2 s k-s} \zeta(2 s k-s)}{2 k-1}-\frac{x^{s k} \zeta(s k)}{k}\right)\right\},
\end{aligned}
$$

with $\Re(s)>1$ in $(11), \Re(s)>0$ in $(12)$, and $\zeta\left(\{\ldots\}_{0}\right)=1$. At $s=1$, generator $(12)$ becomes

$$
A(x) \equiv \sum_{n \geq 0} x^{n} \zeta\left(\{\overline{1}\}_{n}\right)=\frac{2}{B\left(1+\frac{1}{2} x, \frac{1}{2}-\frac{1}{2} x\right)} .
$$

We find, empirically, that cases with alternate alternations of sign are generated by

$$
M(x) \equiv \sum_{n \geq 0}\left\{x^{2 n} \zeta\left(\{\overline{1}, 1\}_{n}\right)+x^{2 n+1} \zeta\left(\{\overline{1}, 1\}_{n}, \overline{1}\right)\right\} \stackrel{?}{=}\left|A\left(\frac{x}{1+i}\right)\right|^{2}
$$

for real $x$. This, in turn, generates (8), via the convolution

$$
\sum_{n \geq 0} x^{4 n} \zeta\left(\{3,1\}_{n}\right) \stackrel{?}{=} M(x) M(-x) .
$$

With a further alternating summation, the result analogous to (14) is

$$
\begin{aligned}
T(x) & \equiv 1+\sum_{n \geq 0}\left\{x^{2 n+1} \zeta\left(\overline{1},\{\overline{1}, 1\}_{n}\right)+x^{2 n+2} \zeta\left(\overline{1},\{\overline{1}, 1\}_{n}, \overline{1}\right)\right\} \\
& \stackrel{?}{=} M(x)\left\{1-x \Im \psi\left(1+\frac{1}{2} \frac{x}{1+i}\right)-x \Im \psi\left(\frac{1}{2}-\frac{1}{2} \frac{x}{1+i}\right)\right\} .
\end{aligned}
$$

Convolution of (16), in the manner of (15), also generates self-dual non-alternating sums:

$$
\sum_{n \geq 0} x^{4 n+2} \zeta\left(2,\{1,3\}_{n}\right) \stackrel{?}{=} 1-T(x) T(-x) .
$$

Moreover, we discovered the remarkable two-parameter self-dual result

$$
\zeta\left(\{2\}_{m},\left\{3,\{2\}_{m}, 1,\{2\}_{m}\right\}_{n}\right) \stackrel{?}{=} \frac{2(m+1) \cdot \pi^{4(m+1) n+2 m}}{(2\{m+1\}\{2 n+1\}) !}
$$


of which the previously known [10] example (8) is the $m=0$ case. David Bailey (personal communication) has confirmed (18) for $1 \leq m, n \leq 4$ to 800 decimal places.

Results for sums with unit exponents are generated by

$$
\begin{aligned}
& L(x) \equiv \sum_{n \geq 0} x^{n} \zeta\left(\overline{1},\{1\}_{n}\right)=\frac{2^{-x}-1}{x}, \\
& \sum_{n \geq 0} x^{n} \zeta\left(\overline{1}, \overline{1},\{1\}_{n}\right)=\sum_{k \geq 1} \frac{2^{-k}}{k(x-k)}, \\
& \sum_{n \geq 0} x^{n} \zeta\left(\overline{1},\{1\}_{n}, \overline{1}\right) \stackrel{?}{=} \sum_{k \geq 1} \frac{L(k+x)}{k}+L(x) \log 2, \\
& \sum_{m, n \geq 0} x^{m+1} y^{n+1} \zeta\left(\overline{1},\{1\}_{m} \overline{1}, \overline{1},\{1\}_{n}\right) \stackrel{?}{=} \sum_{k \geq 1}\{L(k+x)-L(k) \\
&\left.-\frac{L(k+x-y)-L(k-y)}{2^{y}}\right\} .
\end{aligned}
$$

We also discovered the following reductions to non-alternating sums and unit-exponent alternating sums:

$$
\begin{aligned}
\zeta\left(\{\overline{2}, 1\}_{n}\right) \stackrel{?}{=} & 8^{-n} \zeta\left(\{2,1\}_{n}\right)=8^{-n} \zeta\left(\{3\}_{n}\right) \\
\zeta\left(\overline{1},\{1\}_{m}, 2,\{1\}_{n}\right) \stackrel{?}{=} & \zeta\left(\overline{1},\{1\}_{n}, \overline{1}, \overline{1},\{1\}_{m}\right)-\zeta\left(\overline{1},\{1\}_{m+n+2}\right) \\
\zeta\left(\overline{1}, \overline{1},\{1\}_{m}, 2,\{1\}_{n}\right) \stackrel{?}{=} & \zeta\left(\overline{1}, \overline{1},\{1\}_{n}, \overline{1}, \overline{1},\{1\}_{m}\right)-\zeta\left(\overline{1}, \overline{1},\{1\}_{m+n+2}\right) \\
& +\zeta\left(\overline{1}, \overline{1},\{1\}_{m}\right) \zeta(n+2) \\
\zeta\left(\overline{1},\{1\}_{m}, 2,2,\{1\}_{n}\right) \stackrel{?}{=} & \zeta\left(\overline{1},\{1\}_{n}, \overline{1}, \overline{1}, \overline{1}, \overline{1},\{1\}_{m}\right)+\zeta\left(\overline{1},\{1\}_{m+n+4}\right) \\
& -\zeta\left(\overline{1},\{1\}_{n+2}, \overline{1}, \overline{1},\{1\}_{m}\right)-\zeta\left(\overline{1},\{1\}_{n}, \overline{1}, \overline{1},\{1\}_{m+2}\right) \\
\zeta\left(\overline{1}, \overline{1},\{1\}_{m}, 2,2,\{1\}_{n}\right) \stackrel{?}{=} \quad \zeta\left(\overline{1}, \overline{1},\{1\}_{n}, \overline{1}, \overline{1}, \overline{1}, \overline{1},\{1\}_{m}\right)+\zeta\left(\overline{1}, \overline{1},\{1\}_{m+n+4}\right) & \\
& -\zeta\left(\overline{1}, \overline{1},\{1\}_{n+2}, \overline{1}, \overline{1},\{1\}_{m}\right)-\zeta\left(\overline{1}, \overline{1},\{1\}_{n}, \overline{1}, \overline{1},\{1\}_{m+2}\right) \\
& +\zeta\left(\overline{1}, \overline{1},\{1\}_{m}, 2\right) \zeta(n+2) \\
& -\zeta\left(\overline{1}, \overline{1},\{1\}_{m}\right)\{\zeta(n+4)+\zeta(2, n+2)\} \\
\zeta\left(\overline{m+1},\{1\}_{n}\right) \stackrel{?}{=}(-1)^{m} \sum_{k \leq 2^{m}} \varepsilon_{k} \zeta\left(\overline{1},\{1\}_{n}, S_{k}\right) & \stackrel{?}{=}(-1)^{m} \sum_{k \leq 2^{m}} \varepsilon_{k} \zeta\left(\overline{1}, \overline{1},\{1\}_{n}, S_{k}\right) \\
\zeta\left(\overline{1}, \overline{m+1},\{1\}_{n}\right) \stackrel{(27)}{ } &
\end{aligned}
$$


THE ElECTRONiC JOURNAL OF COMBINATORICS 4 (No.2) (1997), \#R5

$$
-\sum_{p \leq m}(-1)^{p} \zeta\left(m-p+2,\{1\}_{n}\right) \zeta(\bar{p})
$$

where the last two involve summation over all $2^{m}$ unit-exponent substrings of length $m$, with $\sigma_{k, j}$ as the $j$ th sign of substring $S_{k}$, and $\varepsilon_{k}=\prod_{m / 2>i \geq 0} \sigma_{k, m-2 i}$, whose effect is to restrict the innermost $m$ summation variables to alternately odd and even integers.

We remark that (11) reduces $(23)$ to zetas, and that $(19,22)$ reduce (24) to zetas and the polylogarithms $L i_{n}(1 / 2)$. The $m=1$ case of (28) is reduced to polylogarithms by $(19,21)$. The product terms in (25) and (29) are reduced by (20) and (10); those in (27) involve terms given by $(20,25)$. The analysis of [4] shows that new irreducibles, beyond the polylogarithms from (19-22), result from unit-exponent terms generated by $(25,26,27)$, by $(28)$ when $m \geq 2$, and by (29) when $m \geq 1$.

\section{Evaluations at arbitrary depth}

From the symmetric generator (10), we obtain

$$
\begin{aligned}
\zeta\left(2,\{1\}_{n}\right) & =\zeta(n+2), \\
\zeta\left(3,\{1\}_{n}\right) & =\zeta(n+2,1)=\frac{n+2}{2} \zeta(n+3)-\frac{1}{2} \sum_{k=1}^{n} \zeta(k+1) \zeta(n+2-k),
\end{aligned}
$$

and, in general, products of up to $\min (m+1, n+1)$ zetas in $\zeta\left(m+2,\{1\}_{n}\right)=\zeta\left(n+2,\{1\}_{m}\right)$, whose symmetry was known from $(7)$. Note that (30) is also implied by $(9)$.

For integer values, $s=m$, generators 11,12 give

$$
\begin{aligned}
\sum_{n \geq 0} x^{m n} \zeta\left(\{m\}_{n}\right) & =\prod_{j=1}^{m} \frac{1}{\Gamma\left(1-\omega_{m}^{2 j-1} x\right)}, \\
\sum_{n \geq 0} x^{m n} \zeta\left(\{\bar{m}\}_{n}\right) & =\prod_{j=1}^{m} \frac{\sqrt{\pi}}{\Gamma\left(1-\frac{1}{2} \omega_{m}^{2 j-1} x\right) \Gamma\left(\frac{1}{2}-\frac{1}{2} \omega_{m}^{2 j} x\right)},
\end{aligned}
$$

with $\omega_{m}=\exp (i \pi / m)$.

For even integers, $m=2 p$, generators $(32,33)$ give trigonometric products:

$$
\begin{aligned}
S_{p}(x) \equiv & \sum_{n \geq 0} x^{2 p n} \zeta\left(\{2 p\}_{n}\right)=(i \pi x)^{-p} \prod_{j=1}^{p} \sin \left(\pi \omega_{2 p}^{2 j-1} x\right), \\
\sum_{n \geq 0} x^{2 p n} \zeta\left(\{\overline{2 p}\}_{n}\right) & =S_{p}\left(\frac{1}{2} x\right) \prod_{j=1}^{p} \cos \left(\frac{1}{2} \pi \omega_{p}^{j} x\right),
\end{aligned}
$$


THE ElECTRONiC JOURNAL OF COMBINATORICS 4 (No.2) (1997), \#R5

which show that $\zeta\left(\{2 p\}_{n}\right)$ and $\zeta\left(\{\overline{2 p}\}_{n}\right)$ are rational multiples of $\pi^{2 p n}$.

The non-alternating result (34) readily yields

$$
\begin{aligned}
\zeta\left(\{2\}_{n}\right) & =\frac{2 \cdot(2 \pi)^{2 n}}{(2 n+1) !}\left(\frac{1}{2}\right)^{2 n+1}, \\
\zeta\left(\{4\}_{n}\right) & =\frac{4 \cdot(2 \pi)^{4 n}}{(4 n+2) !}\left(\frac{1}{2}\right)^{2 n+1}, \\
\zeta\left(\{6\}_{n}\right) & =\frac{6 \cdot(2 \pi)^{6 n}}{(6 n+3) !} \\
\zeta\left(\{8\}_{n}\right) & =\frac{8 \cdot(2 \pi)^{8 n}}{(8 n+4) !}\left\{\left(1+\frac{1}{\sqrt{2}}\right)^{4 n+2}+\left(1-\frac{1}{\sqrt{2}}\right)^{4 n+2}\right\} .
\end{aligned}
$$

Comparison of (37) with (8) reveals that Zagier's conjecture can be reformulated as

$$
4^{n} \zeta\left(\{3,1\}_{n}\right) \stackrel{?}{=} \zeta\left(\{4\}_{n}\right)
$$

or, in the notation of $(5)$,

$$
4^{n} \int_{0}^{1}\left(\Omega^{2} \omega^{2}\right)^{n} \stackrel{?}{=} \int_{0}^{1}\left(\Omega^{3} \omega\right)^{n}
$$

Equivalently, from (36), it becomes

$$
(2 n+1) \zeta\left(\{3,1\}_{n}\right) \stackrel{?}{=} \zeta\left(\{2,2\}_{n}\right)
$$

or

$$
(2 n+1) \int_{0}^{1}\left(\Omega^{2} \omega^{2}\right)^{n} \stackrel{?}{=} \int_{0}^{1}(\Omega \omega)^{2 n},
$$

in which, ynlike (41), the list of omegas is merely reordered. Comparison of the empirical result (18) with $(36,37)$ reveals that

$$
\begin{aligned}
\zeta\left(\{2\}_{m},\left\{3,\{2\}_{m}, 1,\{2\}_{m}\right\}_{n}\right) & \stackrel{?}{=} \frac{1}{2 n+1} \zeta\left(\{2\}_{2(m+1) n+m}\right), \\
\zeta\left(\{2\}_{2 p},\left\{3,\{2\}_{2 p}, 1,\{2\}_{2 p}\right\}_{n}\right) & \stackrel{?}{=} \frac{2 p+1}{4^{(2 p+1) n+p}} \zeta\left(\{4\}_{(2 p+1) n+p}\right) .
\end{aligned}
$$

Result (39) was already known [9]. The next member of the series is rather beautiful:

$$
\zeta\left(\{10\}_{n}\right)=\frac{10 \cdot(2 \pi)^{10 n}\left(L_{10 n+5}+1\right)}{(10 n+5) !},
$$


THE ElECTRONiC JOURNAL OF COMBINATORICS 4 (No.2) (1997), \#R5

where $L_{n}=L_{n-1}+L_{n-2}$ is the $n$th Lucas number, with $L_{1}=1$ and $L_{2}=3$.

In the general case, a Laplace transform of (34) yields

$$
\sum_{n \geq 0}(2 p n+p) !\left(\frac{z}{(2 \pi)^{p}}\right)^{n} \zeta\left(\{2 p\}_{n}\right)=2 p \sum_{k=1}^{N_{p}} \frac{z_{p, k}^{1 / 2}}{z_{p, k}-z}
$$

with $N_{p} \leq 2^{p} / 2 p$ poles, whose positions $\left\{z_{p, k} \mid 1 \leq k \leq N_{p}\right\}$ are determined by the Laplace transforms of the $2^{p}$ exponentials generated by the product in $(34)$. The pole closest to the origin, at $z_{p, 1}=(2 \sin (\pi / 2 p))^{2 p}$, gives the first term in

$$
\zeta\left(\{2 p\}_{n}\right)=\frac{2 p \cdot(2 \pi)^{2 p n}}{(2 p n+p) !}\left(\frac{1}{2 \sin \frac{\pi}{2 p}}\right)^{2 p n+p}\left\{1+\sum_{k=2}^{N_{p}} R_{p, k}^{2 p n+p}\right\},
$$

with $R_{p, k}=\left(z_{p, 1} / z_{p, k}\right)^{1 / 2 p}$, and hence $\left|R_{p, k}\right|<1$ for $k>1$. Choices of signs, $\sigma_{j}= \pm 1$, in

$$
\frac{\left|R_{p, k}\right|}{\sin \frac{\pi}{2 p}}=\left|\sum_{j=1}^{p} \sigma_{j} \omega_{p}^{j}\right|
$$

yield all the absolute values, though some choices of sign may not be realized in (48).

Proceeding up to $p=9$, we derived:

$$
\begin{aligned}
& \zeta\left(\{12\}_{n}\right)=\frac{12 \cdot(2 \pi)^{12 n}}{(12 n+6) !}\left\{\left(\frac{1+\sqrt{3}}{\sqrt{2}}\right)^{12 n+6}+\left(\frac{1-\sqrt{3}}{\sqrt{2}}\right)^{12 n+6}+2^{6 n+3}\right\}, \\
& \zeta\left(\{14\}_{n}\right)=\frac{14 \cdot(2 \pi)^{14 n}}{(14 n+7) !} \Re\left(\sum_{k=1}^{3} \frac{1+r_{k}^{28 n+14}}{\left.r_{k}^{14 n+7}+2\left(\frac{i \sqrt{7}-1}{2}\right)^{14 n+7}+1\right),}\right. \\
& \zeta\left(\{16\}_{n}\right)=\frac{16 \cdot(2 \pi)^{16 n}}{(16 n+8) !} \sum_{k=1}^{4} \Re\left(\frac{1}{s_{k}^{16 n+8}}+\frac{s_{k}^{16 n+8}}{c_{k}^{16 n+8}}+2\left(\frac{i}{c_{k}}+c_{k}+\sqrt{2}\right)^{8 n+4}\right), \\
& \zeta\left(\{18\}_{n}\right)=\frac{18 \cdot(2 \pi)^{18 n}}{(18 n+9) !} \sum_{k=1}^{3} \Re\left(\frac{1}{t_{k}^{18 n+9}}+\left(1+t_{k}\right)^{18 n+9}+2\left(-\omega_{3}-t_{k}\right)^{18 n+9}\right) .
\end{aligned}
$$

In $(51), r_{k}=2 \cos ((2 k-1) \pi / 7)$ are the roots of the cubic equation $r(1+r)(2-r)=1$. In $(52), s_{k}=2 \sin ((2 k-1) \pi / 16)$ and $c_{k}=2-s_{k}^{2}$, which are the roots of $\left(2-c^{2}\right)^{2}=2$. In $(53), t_{k}=2 \cos \left(2^{k} \pi / 9\right)$ are the roots of $t\left(3-t^{2}\right)=1$. The method adopted to obtain these results exploited the exactness of the $[N-1 \backslash N]$ Padé approximant to (47), for $N \geq N_{p}$. The roots of its denominator were then used to find $R_{p, k}=2 \sin (\pi / 2 p) / z_{p, k}^{1 / 2 p}$. 
THE ElECTRONiC JOURNAL OF COMBINATORICS 4 (No.2) (1997), \#R5

The $p$-th member of the integer sequence ${ }^{2}$

$1,1,1,2,3,4,8,12,16,33,62,67,186,316,280,1040,1963,1702,6830,10751, \ldots$

gives the number of distinct non-zero absolute values of $\sum_{j=1}^{p} \sigma_{j} \omega_{p}^{j}$. Of these possibilities,

$$
1,1,1,2,3,3,8,12,9, \ldots
$$

are present in (48). Hence, for $p=6$ and $p=9$, some of the choices of signs in (49) are absent. Correspondingly, the values of $N_{p}$ in the sequence

$$
1,1,1,2,3,3,9,16,12, \ldots
$$

do not saturate the upper bound $\left\lfloor 2^{p} / 2 p\right\rfloor$, for $p=6$ and $p=9$.

Explicit results from (35) are much lengthier than those from $(34)$ since the former gives $4^{p}$ exponentials, while the latter gives only $2^{p}$. We cite only the first three cases:

$$
\begin{aligned}
\zeta\left(\{\overline{2}\}_{n}\right)= & \frac{\pi^{2 n}}{(2 n+1) !} \frac{(-1)^{n(n+1) / 2}}{2^{n}}, \\
\zeta\left(\{\overline{4}\}_{n}\right)= & \frac{\pi^{4 n}}{(4 n+2) !} \frac{(-1)^{n(n+1) / 2}}{2^{n}}\left((1+\sqrt{2})^{2 n+1}+(1-\sqrt{2})^{2 n+1}\right), \\
\zeta\left(\{\overline{6}\}_{n}\right)= & \frac{\pi^{6 n}}{(6 n+3) !} \cdot \frac{3}{2}\left(1+2^{3 n+1}(-1)^{n(n+1) / 2}\right. \\
& \left.\quad \times\left\{\left(\frac{1+\sqrt{3}}{2}\right)^{6 n+3}+\left(\frac{1-\sqrt{3}}{2}\right)^{6 n+3}-1\right\}\right) .
\end{aligned}
$$

Comparison of (36) with (57) reveals that

$$
\zeta\left(\{\overline{2}\}_{n}\right)=2^{-n}(-1)^{\lceil n / 2\rceil} \zeta\left(\{2\}_{n}\right) .
$$

Finally, from (12) we obtain

$$
\zeta\left(\{\overline{1}\}_{n}\right)=(-1)^{n} \sum \prod_{k \geq 1} \frac{1}{j_{k} !}\left(\frac{-L i_{k}\left((-1)^{k}\right)}{k}\right)^{j_{k}}
$$

where the sum is over all non-negative integers satisfying $\sum_{k \geq 1} k j_{k}=n$.

\footnotetext{
${ }^{2}$ The integer sequence (54) was not identified by Neil Sloane's 'superseeker' utility [19].
} 
THE ElECTRONiC JOURNAL OF COMBINATORICS 4 (No.2) (1997), \#R5

From (17) we obtain a self-dual evaluation, more complex than (18):

$$
\begin{aligned}
\zeta\left(2,\{1,3\}_{n}\right) \stackrel{?}{=} & 4^{-n} \sum_{k=0}^{n}(-1)^{k} \zeta\left(\{4\}_{n-k}\right)\{(4 k+1) \zeta(4 k+2) \\
& \left.-4 \sum_{j=1}^{k} \zeta(4 j-1) \zeta(4 k-4 j+3)\right\},
\end{aligned}
$$

with $\pi^{2}$ terms generated by $\zeta(4 k+2)$ and by (37). The absence of $\zeta(4 k+1)$ is conspicuous.

Explicit results generated by $(19-22)$ involve the polylogarithms

$$
A_{n} \equiv L i_{n}(1 / 2)=\sum_{k=1}^{\infty} \frac{1}{2^{k} k^{n}}, \quad P_{n} \equiv \frac{(\ln 2)^{n}}{n !}, \quad Z_{n} \equiv(-1)^{n} \zeta(n)
$$

in terms of which we obtain

$$
\begin{aligned}
\zeta\left(\overline{1},\{1\}_{n}\right) & =(-1)^{n+1} P_{n+1} \\
\zeta\left(\overline{1}, \overline{1},\{1\}_{n}\right) & =-A_{n+2} \\
\zeta\left(\overline{1},\{1\}_{n}, \overline{1}\right) & \stackrel{?}{=}-Z_{n+2}+(-1)^{n} \sum_{k=1}^{n+2} A_{k} P_{n+2-k} \\
\zeta\left(\overline{1},\{1\}_{m}, \overline{1}, \overline{1},\{1\}_{n}\right) & \stackrel{?}{=}(-1)^{m} \sum_{k=1}^{m+2}\left(\begin{array}{c}
n+k \\
n+1
\end{array}\right) A_{k+n+1} P_{m+2-k} \\
& +(-1)^{n} \sum_{k=1}^{n+2}\left(\begin{array}{c}
m+k \\
m+1
\end{array}\right) Z_{k+m+1} P_{n+2-k} .
\end{aligned}
$$

We also have

$$
\zeta\left(\overline{2},\{1\}_{n}\right)=-Z_{n+2}+2(-1)^{n+1} P_{n+2}+(-1)^{n} \sum_{k=0}^{n+2} A_{k} P_{n+2-k},
$$

which shows that (67) and the $m=1$ case of (28) are equivalent. The complexity of the proof of (69), outlined in the Appendix, may serve as an indication of the difficulty of proving (28) in general.

\section{Evaluations at specific depths}

Several thousand evaluations, obtained in the work for [4] with the aid of MPPSLQ [1] and REDUCE [15] were inspected, in a search for further, comparably simple, results. These 
include analytical results for all 1457 sums with weight $w=\sum_{j} s_{j} \leq 7$, for all 3698 double sums with weight $w \leq 44$, and for all 1092 non-alternating sums with depth $k \leq 4$ and weight $w \leq 14$. To these we adjoined more than 2000 strategically selected high-precision numerical evaluations of self-dual sums with $s_{j} \leq 3$ and weights up to $w=40$, which enabled the discovery and validation of the remarkable generalization of (8) that is given in (18) The reader will find a detailed discussion of our scheme for computing these highprecision numerical evaluations in section 4 of [4]. For other approaches, see [12] and [11] in which Euler-Maclaurin based techniques are eschewed in favour of transtormation to explicitly convergent sums.

It was found that precisely 11 of the 64 convergent depth- 7 sums with unit exponents are reduct the polylogarithms (64) and their products. They are given by the 6 results $13,14,16$ 65,66,67) and 5 instances of (68). Combining these with 5 instances of (24) and the $m=1$ case of (28), we exhaust the weight-7 reducible alternating sums with depth $k \geq 5$. We computed, to high precision, all 2046 self-dual non-alternating sums comprising up to 10 'atomic' substrings of the form $\left\{m+2,\{1\}_{n}\right\}$, with $m, n=0,1$, as in $(18, \$ 3)$ and hence having weight $w=2 k \leq 40$. Precisely 25 of these are rational multiples of powers of $\pi^{2}$. They are exhausted by (18) Moreover, $(10,18,63)$ were found to exhaust all zeta-reducible cases of non-alternating sums with $w=2 k=10$, of self-dual sums with $w=12$, and of self-dual sums with $s_{j} \leq 3$ and $8 \leq w \leq 16$. At $w=16$, computation and MPPSLQ analysis of 34 self-dual sums, to 300 significant figures, took about 0.5 CPUhour/sum on a DEC AlphaStation 600 5/333 at the Open University. Such exhaustion of reducible cases by our results (10-29) suggests that they are, like our database, reasonably comprehensive.

Among many MPPSLQ results at specific depths, the following are rather distinctive:

$$
\begin{aligned}
\zeta(2,1, \overline{2}, \overline{2}) \stackrel{?}{=} & \frac{39}{128} \zeta(4) \zeta(3)-\frac{193}{64} \zeta(5) \zeta(2)+\frac{593}{128} \zeta(7), \\
\zeta(\overline{2}, \overline{2}, 1,2) \stackrel{?}{=} & \frac{9}{128} \zeta(4) \zeta(3)+\frac{447}{128} \zeta(5) \zeta(2)-\frac{1537}{256} \zeta(7), \\
\zeta\left(\{4,1,1\}_{2}\right) \stackrel{?}{=} & \frac{3 \pi^{4}}{16}\{\zeta(6,2)-4 \zeta(5) \zeta(3)\}-\frac{41 \pi^{6}}{5040}\left\{\zeta^{2}(3)-\frac{77023 \pi^{6}}{14414400}\right\} \\
& +\frac{397}{8} \zeta(9) \zeta(3)+\zeta^{4}(3), \\
\zeta(2,2,1,2,3,2) \stackrel{?}{=} & \frac{75 \pi^{2}}{32}\left\{\zeta(8,2)-2 \zeta(7) \zeta(3)+\frac{34}{225} \zeta^{2}(5)+\frac{4528801 \pi^{10}}{61297236000}\right\} \\
& -\frac{825}{8} \zeta(7) \zeta(5), \\
\zeta\left(\{\overline{3}, 1\}_{2}\right) \stackrel{?}{=} & -7\left(\alpha(5)-\frac{39}{64} \zeta(5)+\frac{1}{8} \zeta(4) \ln 2\right) \zeta(3)+\left(2 \alpha(4)-\frac{1}{4} \zeta(4)\right)^{2} \\
& +2\left(\alpha(4)-\frac{15}{16} \zeta(4)+\frac{7}{8} \zeta(3) \ln 2\right)^{2}-\frac{1}{32} \zeta(8),
\end{aligned}
$$


with $\alpha(n) \equiv A_{n}+(-1)^{n}\left(P_{n}-\frac{\pi^{2}}{12} P_{n-2}\right)$, as in $[4]$. Note that the alternating sums $(70,71)$ are pure zeta, yet we were unable to find generalizations of them; only from (12,23) have we obtained arbitrary-depth pure-zeta alternating results. Note also that the selfdual sums $(72)$ and $(73)$, with $w=2 k=12$, contain non-zeta [2] irreducibles, $\zeta(6,2)$ and $\zeta(8,2)$, yet their kinship with distinct reducible classes, generated by (15) and (17) manifests itself in the unusual circumstance that they share only $\pi^{12}$ as a common Finally, note that the polylogarithmic complexity of (74) contrasts greatly with the zetareducibility of (23), via (11), yet its kinship with (23) is reflected by the absence of 12 of the 21 terms [4] that occur in alternating sums with $w=2 k=8$. In each of (7074) one senses, from the relatively small number of terms, a degree of proximity to an arbitrary-depth reduction.

It is conjectured that, at any depth $k>1$, Euler sums of weight $w$ are reducible to a rational linear combination of lesser-depth sums (and their products) whenever $w$ and $k$ are of opposite parity. It is also conjectured that the lowest-weight irreducible depth- $k$ alternating sum occurs at weight $k+2$ and entails $L i_{k+2}(1 / 2)[4]$. The critical weight $w_{k}$, at which depth- $k$ non-alternating sums first fail to be reducible to non-alternating sums of lesser depth, is more problematic. In [2] it was found that $w_{2}=8$; in [3] that $w_{3}=11$; in [4] that $w_{4}=12$. Reducibility was ploved below these critical weights; reducibility at them was shown to be incredible, by lattice methods [1]. There is likewise good support for $w_{5}=15$ and $w_{6}=18$. It is conjectured [5] that $w_{k}=3 k$, for all $k \geq 4$. It appears that a large majority of non-alternating sums are irreducible whenever $w$ and $k$ are of the same parity and $w \geq w_{k}$. Additionally, R. Girgensohn (personal communication) has outlined a proof that, in the notation of (1).

$$
\zeta\left(s_{1}, \ldots, s_{k} ; \sigma_{1}, \ldots, \sigma_{k}\right)+(-1)^{k} \zeta\left(s_{k}, \ldots, s_{1} ; \sigma_{k}, \ldots, \sigma_{1}\right)
$$

is reducible for every $k>1$.

For depths 2, 3 and 4, we have the following more specific remarks:

Depth 2. Whenever $s+t$ is odd, we have

$$
\zeta(s, t ; \sigma, \tau)=\frac{1}{2}\left(-\lambda_{s+t}+\left(1+(-1)^{s}\right) \zeta(s ; \sigma) \zeta(t ; \tau)+\mu_{s+t}\right)-\sum_{0<k<(s+t) / 2} \lambda_{2 k} \mu_{s+t-2 k},
$$

where $\lambda_{r}=\zeta(r ; \sigma \tau)$ and $\mu_{r}=(-1)^{s}\left(\left(\begin{array}{c}r-1 \\ s-1\end{array}\right) \zeta(r ; \sigma)+\left(\begin{array}{c}r-1 \\ t-1\end{array}\right) \zeta(r ; \tau)\right)$. This compact formula summarizes the evaluations given in [3]. Recently, a shorter proof has been given by R. Girgensohn (personal communication). A conjectured minimal Q-basis for all depth-2 
Euler sums is formed by [4]: the depth-1 sums, $\ln 2, \pi^{2},\{\zeta(2 a+1) \mid a>0\}$, and the depth2 sums $\{\zeta(\overline{2 a+1}, \overline{2 b+1)} \mid a>b \geq 0\}$. All 3698 convergent double sums with weights $w \leq 44$ have been proved [4] to be expressible in this basis, using identities derived in [2] and augmented in [4]. A conjectured minimal Q-basis for non-alternating depth-2 Euler sums is formed by $\pi^{2},\{\zeta(2 a+1) \mid a>0\}$ and $\{\zeta(2 a+1,2 b+1) \mid a \geq 2 b>0\}$, which is likewise proven to be sufficient up to weight 44. It is conjectured that the proven result [2]

$$
\zeta(4,2)=\zeta^{2}(3)-\frac{4 \pi^{6}}{2835}
$$

is the sole case of an even-weight reduction of a non-alternating $\operatorname{sum} \zeta(a, b)$ with $a>b>1$.

Depth 3. In [3], it is proved that non-alternating Euler sums of depth 3 and weight $w$ are reducible to a rational linear combination of lesser depth sums when $w$ is even or $w \leq 10$. It is conjectured that most depth-3 non-alternating sums of odd weight exceeding 10 are irreducible. The only reductions that have been found at odd weights in the range 17 to 33 are the cases $\zeta(a, a, a)$ and $\zeta(a, 1,1)$. A conjectured Q-basis for all depth-3 non-alternating sums is the set of lesser-depth non-alternating sums along with the set $\{\zeta(2 a+1,2 b+1,2 c+1) \mid a \geq b \geq c>0, a>c\}$.

Depth 4. It is proved [5] that every depth-4 non-alternating Euler sum with weight less than 12 is reducible to non-alternating sums of lesser depth. It is conjectured that a depth-4 non-alternating Euler sum with even weight exceeding 14 is reducible if and only if it is of one of the following forms: $\zeta(a, b, a, 1), \zeta(a, a, 1, b), \zeta(a, 1, b, b)$, or $\zeta(a, b, b, a)$, with $a=b$, or $b=1$, permitted. (It is proven and will be shown in a subsequent paper that these forms reduce.)

For more on questions of reducibility, see $[4,5]$.

\section{Conclusions}

Euler sums of arbitrary depth are a rich source of fascinating identities, with (16) and (18) serving as spectacular examples. Many of our results were discovered empirically; to date, we have not proven conjectures (14-18, 21-29) and their corollaries. The evidence in their favour is, however, overwhelming. The reader may consult the appendix for sketched derivations of results that have been proved.

Acknowledgements. We thank Dirk Kreimer for informing us that (7) s in [17]. Richard 
THE ELECTROniC Journal of COMBinatorics 4 (No.2) (1997), \#R5

Crandall for telling us about (8) and (39), and Chris Stoddart for skillful computer management.

\section{Appendix: Some Proof Sketches}

The integral representation (2) may be derived using the well-known identity

$$
n^{-s} \Gamma(s)=\int_{1}^{\infty}(\log y)^{s-1} y^{-n-1} d y
$$

Thus, the LHS of (2) may be written as

$$
\zeta\left(s_{1}, \ldots, s_{k} ; \sigma_{1}, \ldots, \sigma_{k}\right)=\sum \prod_{j=1}^{k} \int_{1}^{\infty} \frac{d y_{j}}{y_{j}} \frac{\left(\log y_{j}\right)^{s_{j}-1}}{\Gamma\left(s_{j}\right)}\left(\frac{\sigma_{j}}{y_{j}}\right)^{n_{j}},
$$

where the sum is over all positive integers $n_{1}>n_{2}>\cdots>n_{k}>0$. Now make the change of summation variables $m_{k}=n_{k}$, and $m_{j}=n_{j}-n_{j+1}$ for $j=1,2, \ldots, k-1$. Then each $m_{j}$ runs independently over the positive integers, and (78) becomes

$$
\begin{aligned}
\zeta\left(s_{1}, \ldots, s_{k} ; \sigma_{1}, \ldots, \sigma_{k}\right) & =\prod_{j=1}^{k} \int_{1}^{\infty} \frac{d y_{j}}{y_{j}} \frac{\left(\log y_{j}\right)^{s_{j}-1}}{\Gamma\left(s_{j}\right)} \sum_{m_{j} \geq 1}\left(\prod_{i=1}^{j} \frac{\sigma_{i}}{y_{i}}\right)^{m_{j}} \\
& =\prod_{j=1}^{k} \frac{1}{\Gamma\left(s_{j}\right)} \int_{1}^{\infty} \frac{d y_{j}}{y_{j}} \frac{\left(\log y_{j}\right)^{s_{j}-1}}{\prod_{i=1}^{j} y_{i} / \sigma_{i}-1},
\end{aligned}
$$

after summing the geometric series. Since each $\sigma_{i}= \pm 1$, this is the same as (2).

In the introduction, we briefly indicated how the iterated-integral representation $(5)$ arises from the non-iterated multiple integral representation (2). We present a direct derivation below. Yet another approach is taken in [17], but there only the non-alternating case is considered. With $\Omega$ and $\omega_{j}$ as in the introduction, put $\Omega_{n}:=x^{n} \Omega=x^{n} d x / x$. We begin with the self-evident integral representation

$$
\frac{y^{n}}{n^{k}}=\int_{0}^{y} \Omega^{k-1} \Omega_{n}
$$

valid for positive integers $n$ and $k$. It follows that for positive integers $n, p, r$, and $k$,

$$
\frac{y^{n+p}}{(n+p)^{r} n^{k}}=\frac{1}{n^{k}} \int_{0}^{y} \Omega^{r-1} \Omega_{n+p}=\int_{0}^{y} \Omega^{r-1}\left(\frac{x^{n}}{n^{k}}\right) x^{p} \frac{d x}{x}
$$


THE ELECTRONiC JOURNAL OF COMBINATORICS 4 (No.2) (1997), \#R5

Now substitute (80) for $x^{n} / n^{k}$, obtaining

$$
\frac{y^{n}}{n^{r}(n+p)^{k}}=\int_{0}^{y} \Omega^{r-1} \int_{0}^{x} \Omega^{k-1} \Omega_{n} x^{p} \frac{d x}{x}=\int_{0}^{y} \Omega^{r-1} \Omega_{p} \Omega^{k-1} \Omega_{n} .
$$

In general, for positive integers $m_{j}, s_{j}$, we have

$$
\frac{y^{m_{1}}}{\prod_{j=1}^{k}\left(m_{1}+m_{2}+\cdots+m_{j}\right)^{s_{j}}}=\int_{0}^{y} \prod_{j=1}^{k} \Omega^{s_{j}-1} \Omega_{m_{j}} .
$$

But, recalling the definition (4) of $\tau_{j}$ from the introduction, we have

$$
\zeta\left(s_{1}, \ldots, s_{k} ; \sigma_{1}, \ldots, \sigma_{k}\right)=\sum_{n_{j}>n_{j+1}} \prod_{j=1}^{k} \frac{\sigma_{j}^{n_{j}}}{n_{j}^{s_{j}}}=\sum_{m_{j} \geq 1} \prod_{j=1}^{k} \frac{\tau_{j}^{m_{j}}}{\left(m_{1}+m_{2}+\cdots+m_{j}\right)^{s_{j}}} .
$$

Thus, from (83),

$$
\zeta\left(s_{1}, \ldots, s_{k} ; \sigma_{1}, \ldots, \sigma_{k}\right)=\sum_{m_{j} \geq 1} \int_{0}^{1} \prod_{j=1}^{k} \Omega^{s_{j}-1} \tau_{j}^{m_{j}} \Omega_{m_{j}}=\int_{0}^{1} \prod_{j=1}^{k} \Omega^{s_{j}-1} \omega_{j},
$$

by summing the $k$ geometric series and recalling the definition (6) of $\omega_{j}$ from the introduction.

A general property of iterated integrals $[17]$ such as (5) or (85) is that the string in the integrand can be reversed if the integration limits are exchanged and the appropriate sign factor is taken into account. If in addition, the integration variables $x_{j}$ are all replaced by their complement $1-x_{j}$, this has the effect of switching $\Omega$ and $\omega$. Thus,

$$
\begin{aligned}
\zeta\left(m_{1}+2,\{1\}_{n_{1}}, \ldots, m_{p}+2,\{1\}_{n_{p}}\right) & =\int_{0}^{1} \Omega^{m_{1}+1} \omega^{n_{1}+1} \cdots \Omega^{m_{p}+1} \omega^{n_{p}+1} \\
& =\int_{0}^{1} \Omega^{n_{p}+1} \omega^{m_{p}+1} \cdots \Omega^{m_{1}+1} \omega^{n_{1}+1} \\
& =\zeta\left(n_{p}+2,\{1\}_{m_{p}}, \ldots, n_{1}+2,\{1\}_{m_{1}}\right),
\end{aligned}
$$

which proves the duality relation $(7)$

To prove (10), we write the left side as

$$
x y \sum_{m \geq 0} \sum_{k \geq 1} \frac{x^{m}}{k^{m+2}} \prod_{j=1}^{k-1}\left(1+\frac{y}{j}\right) .
$$


After summing on $m$, what remains is an instance of the hypergeometric series with first term omitted:

$$
1-{ }_{2} F_{1}(-x, y ; 1-x)=1-\frac{\Gamma(1-x) \Gamma(1-y)}{\Gamma(1-x-y)}, \quad \Re(x+y)<1 .
$$

To complete the proof, write $\Gamma$ in the form $\exp \left(\int \Gamma^{\prime} / \Gamma\right)$ and employ the Maclaurin series representation for $\Gamma^{\prime} / \Gamma$.

For (11), write

$$
F(x):=\sum_{n \geq 0} x^{s n} \zeta\left(\{s\}_{n}\right)
$$

and note that

$$
F(x)=\prod_{j \geq 1}\left(1+\frac{x^{s}}{j^{s}}\right)
$$

follows directly from the definition (1) Taking the logarithmic derivative, we have

$$
\frac{F^{\prime}}{F}(x)=\sum_{j \geq 1} \frac{s x^{s-1} / j^{s}}{\left(1+x^{s} / j^{s}\right)}
$$

Now expand the denominator of (91) in powers of $x^{s} / j^{s}$ and interchange summation order, obtaining

$$
\frac{F^{\prime}}{F}(x)=\sum_{k \geq 1}(-1)^{k-1} s x^{s k-1} \zeta(s k) .
$$

Finally, integrate, exponentiate, and check that the result agrees with (90) at $x=0$.

The proof of (12) is analogous, with

$$
G(x):=\prod_{j \geq 1}\left(1+(-1)^{j} \frac{x^{s}}{j^{s}}\right)
$$

replacing $F(x)$ in (90) above. Note that the special case (13) is example 1, page 259 of $[20]$.

Although we currently have no proof of (14), from

$$
\begin{aligned}
A\left(\frac{x}{1+i}\right) A\left(\frac{x}{1-i}\right) & =\prod_{j \geq 1}\left(1+\frac{(-1)^{j} x}{j \sqrt{2}} e^{i \pi / 4}\right)\left(1+\frac{(-1)^{j} x}{j \sqrt{2}} e^{-i \pi / 4}\right) \\
& =\prod_{j \geq 1}\left(1+\frac{(-1)^{j} x}{j}+\frac{x^{2}}{2 j^{2}}\right),
\end{aligned}
$$


THE ELECTRONIC JOURNAL OF COMBINATORICS 4 (No.2) (1997), \#R5

it follows that

$$
\left(\frac{1}{2 i}\right)^{n} \sum_{k=0}^{2 n} i^{k} \zeta\left(\{\overline{1}\}_{k}\right) \zeta\left(\{\overline{1}\}_{2 n-k}\right)=\sum_{2 p+q=2 n} \zeta\left(\{\overline{1}\}_{q}\right) 2^{-p} \zeta\left(\{2\}_{p}\right) .
$$

Similarly, from

$$
\prod_{j \geq 1}\left(1+\frac{(-1)^{j} x^{3}}{j^{3}}\right)=\prod_{j \geq 1}\left(1+\frac{(-1)^{j} x}{j}\right)\left(1-\frac{(-1)^{j} x}{j}+\frac{x^{2}}{j^{2}}\right),
$$

it follows that

$$
\zeta\left(\{\overline{3}\}_{n}\right)=\sum_{2 p+q+r=3 n}(-1)^{q} \zeta\left(\{\overline{1}\}_{q}\right) \zeta\left(\{\overline{1}\}_{r}\right) \zeta\left(\{2\}_{p}\right) .
$$

To prove (19) take $t=-1$ in

$$
\sum_{m \geq 1} \frac{t^{m}}{m} \prod_{j=1}^{m-1}\left(1+\frac{x}{j}\right)=t \sum_{m \geq 0}(-t)^{m}\left(\begin{array}{c}
-x-1 \\
m
\end{array}\right) \int_{0}^{1} u^{m} d u=\frac{(1-t)^{-x}-1}{x} .
$$

For (20), consider

$$
S:=\sum_{m \geq 1} \frac{(-1)^{m}}{m} \sum_{k=1}^{m-1} \frac{(-1)^{k}}{k} \prod_{j=1}^{k-1}\left(1+\frac{x}{j}\right)
$$

the generating function for $\zeta\left(\overline{1}, \overline{1},\{1\}_{n}\right)$. Since the inner sum of (100) is the generating function for $\zeta\left(\overline{1},\{1\}_{n}\right)$, we may write, in view of (99),

$$
S=\int_{0}^{-1} \frac{(1+u)^{-x}-1}{x(1-u)} d u=\frac{1}{2} \int_{0}^{1} \frac{1-u^{-x}}{x(1-u / 2)} d u=\sum_{k \geq 1} 2^{-k}\left(\frac{1}{k}-\frac{1}{k-x}\right),
$$

which is the right side of (20).

We factored the generating function (11) into linear factors and then applied the infinite product representation for the Gamma function to arrive at (32). In the same way, we arrived at (33) from (12). The same procedure is done, in greater generality and with more detalls provided, in 20], pp. 238-239. Equations (34) and (35) arise from applying the reflection formula for the Gamma function to (32) and (33) respectively. Evaluations (36) through (39), and (46) were derived from (34) using the addition formulae 
to combine products of sine functions into sums of trigonometric functions. Likewise, evaluations (57) through (60) were derived from (35)

Finally, the promised proof outline of (69) is given. Note that in terms of generating functions, it is equivalent to prove that

$$
\sum_{n \geq 0} t^{n+2}(-1)^{n+1} \zeta\left(\overline{2},\{1\}_{n}\right)=-t(\psi(1-t)+\gamma)+2 \cdot 2^{t}-A(t) 2^{t}-1
$$

where

$$
\begin{aligned}
A(t) & :=\sum_{k \geq 0} t^{k} A(k)=1+\sum_{k \geq 1} \frac{t^{k}(-1)^{k-1}}{(k-1) !} \int_{0}^{1} \log ^{k-1}(1-u) \frac{d u}{1+u} \\
& =1+t \int_{0}^{1} \frac{(1-u)^{-t}}{1+u} d u
\end{aligned}
$$

$\psi$ denotes the logarithmic derivative of the gamma function, and $\gamma$ is Euler's constant. Since

$$
\psi(1-t)+\gamma=\int_{0}^{1} \frac{1-u^{-t}}{1-u} d u, \quad t<1
$$

we need to show that

$$
\int_{0}^{1}\left(\frac{1-u}{2}\right)^{-t} \frac{d u}{1+u}+\int_{0}^{1} \frac{1-u^{-t}}{1-u} d u+\sum_{n \geq 0}(-t)^{n+1} \zeta\left(\overline{2},\{1\}_{n}\right)=\frac{2^{t}-1}{t}
$$

for $|t|<1$ say. But

$$
\begin{aligned}
\sum_{n \geq 0}(-t)^{n+1} \zeta\left(\overline{2},\{1\}_{n}\right) & =-t \sum_{m \geq 0} \frac{(-1)^{m-1}}{(m+1)^{2}} \prod_{j=1}^{m}(1-t / j) \\
& =t \sum_{m \geq 0}\left(\begin{array}{c}
t-1 \\
m
\end{array}\right) \int_{0}^{\infty} y e^{-(m+1) y} d y \\
& =t \int_{0}^{\infty}\left(1+e^{-y}\right)^{t-1} y e^{-y} d y \\
& =-t \int_{0}^{1}(1+u)^{t-1} \log u d u \\
& =\int_{0}^{1} \frac{(1+u)^{t}-1}{u} d u
\end{aligned}
$$


THE ELECTROniC Journal of COMBinatorics 4 (No.2) (1997), \#R5

Therefore, we need only show that

$$
\int_{0}^{1}\left(\frac{1-u}{2}\right)^{-t} \frac{d u}{1+u}+\int_{0}^{1} \frac{1-u^{-t}}{1-u} d u+\int_{0}^{1} \frac{(1+u)^{t}-1}{u} d u=\frac{2^{t}-1}{t}
$$

for suitable $t$. With help from David Borwein, we let $v=(1-u) / 2$ in the first integral, and let $v=1 /(1+u)$ in the third integral. Then the left side of (108) becomes

$$
\begin{aligned}
& \int_{0}^{1 / 2} \frac{v^{-t}}{1-v} d v+\int_{0}^{1 / 2} \frac{1-v^{-t}}{1-v} d v+\int_{1 / 2}^{1} \frac{1-v^{-t}}{1-v} d v+\int_{1 / 2}^{1} \frac{v^{-t}-1}{v(1-v)} d v \\
= & \int_{0}^{1 / 2} \frac{d v}{1-v}+\int_{1 / 2}^{1} \frac{v\left(1-v^{-t}\right)+v^{-t}-1}{v(1-v)} d v \\
= & \int_{1 / 2}^{1} \frac{v^{-t}(1-v)+v-1+1-v}{v(1-v)} d v \\
= & \int_{1 / 2}^{1} v^{-t-1} d v \\
= & \frac{2^{t}-1}{t}
\end{aligned}
$$

as required.

\section{References}

[1] D. H. Bailey, ACM Trans. Math. Software 21 (1995) 379.

[2] D. Borwein, J. M. Borwein and R. Girgensohn, Proc. Edin. Math. Soc. 38 (1995) 273.

[3] J. M. Borwein and R. Girgensohn, CECM research report 95-053 (1995), Electronic J. Combinatorics 3 (1996) R23, with an appendix by D. J. Broadhurst.

[4] D. J. Broadhurst, Open University preprint OUT-4102-62 (1996), hep-th/9604128, to appear in J. Math. Phys.

[5] D. J. Broadhurst, Open University preprint OUT-4102-65 (1996).

[6] D. J. Broadhurst, R. Delbourgo and D. Kreimer, Phys. Lett. B366 (1996) 421. 
THE ELECTROniC Journal of COMBinatorics 4 (No.2) (1997), \#R5

[7] D. J. Broadhurst, J. A. Gracey and D. Kreimer, Open University preprint OUT-4102-46 (1996), hep-th/9607174, to appear in Z. Phys. C.

[8] D. J. Broadhurst and D. Kreimer, Int. J. Mod. Phys. C6 (1995) 519;

Open University preprint OUT-4102-64 (1996), hep-th/9609128.

[9] R. E. Crandall, personal communication.

[10] R. E. Crandall, Topics in advanced scientific computation (Springer, New York, 1995).

[11] R. E. Crandall, Fast numerical evaluation of multiple zeta sums, preprint available from the author at the Center for Advanced Computation, Reed College, Portland, OR.

[12] R. Crandall, J. Buhler, Experimental Mathematics 3,4 (1995) 275-285.

[13] L. Euler, Novi Comm. Acad. Sci. Petropol. 20 (1775) 140.

[14] A. Granville, University of Georgia report.

[15] A. C. Hearn, REDUCE User's Manual Version 3.5, Rand Publication CP78 (1993).

[16] M. E. Hoffman, The algebra of multiple harmonic series, preprint available from the author at the Mathematics Department of the U.S. Naval Academy, Annapolis, MD 21402 .

[17] C. Kassel, Quantum groups (Springer, New York, 1995);

T.Q.T. Le and J. Murakami, MPI Bonn preprints 93-26; 93-89 (1993).

[18] D. Kreimer, Phys. Lett. B354 (1995) 117;

Habilitationsschrift: Renormalization and Knot Theory, Mainz preprint MZ-TH-96-18, q-alg/9607022, to appear in J. Knot Theory and its Ramifications; Knots and Feynman Diagrams (Cambridge University Press, in preparation).

[19] N. J. A. Sloane, Electronic J. Combinatorics 1 (1994) F1.

[20] E. T. Whittaker, G. N. Watson, A Course of Modern Analysis, Cambridge University Press, 4th ed., London, 1969.

[21] D. Zagier, Values of zeta functions and their applications, First European Congress of Mathematics, Volume II, Birkhäuser, Boston, 1994, pp. 497-512. 
THE ElECtronic Journal of COMBINATORICS 4 (No.2) (1997), \#R5

[22] D. Zagier, Multiple Zeta Values, manuscript in preparation. 